\title{
Formas y Estilos de la cerámica prehispánica en Pereira-Risaralda
}

\author{
Shapes and styles of pre-Hispanic ceramics in Pereira-Risaralda \\ Carlos Andrés Jiménez Urueña \\ Facultad de Ciencias ambientales, Instituto Municipal de Cultura y Fomento al Turismo de Pereira y \\ Universidad Tecnológica de Pereira, Pereira, Colombia \\ Correo-e: carljimenezu@gmail.com
}

\begin{abstract}
Resumen- Esta investigación arqueológica se realiza en el marco del proyecto de tesis de grado de antropología del autor y tuvo como objetivo realizar una clasificación del material cerámico que se encuentra en el Instituto Municipal de Cultura y Fomento al Turismo (IMCFT) de la ciudad de Pereira (Risaralda). Con este estudio se determinó las formas y estilos correspondientes a las tipologías cerámicas del Cauca Medio relacionadas con la diversidad de culturas en el tiempo y su distribución espacial. Por otro lado, comparar las diferentes clasificaciones que se ha hecho de la cerámica en esta región con la intención expresa de contextualizar la cerámica. De este modo, caracterizar el tipo de cerámica encontrada en Pereira (Risaralda).
\end{abstract}

Palabras clave - Arqueológica, cerámica prehispánica, formas, estilos, tipologías.

Abstract-- This archaeological research is conducted under the project of anthropology thesis of the author and aimed to classify the ceramic material is at the Municipal Institute of Culture and Tourism Development (IMCFT) of the city of Pereira (Risaralda). This study forms and styles corresponding to the Middle Cauca ceramics typologies related to the diversity of cultures in time and spatial distribution was determined. On the other hand, compare the different classifications made of ceramics in the region with the express intention of contextualizing ceramics. Thus, characterizing the type of pottery found in Pereira (Risaralda).

Key Word - Archaeological, pre-Hispanic ceramics, shapes, styles, types.

\section{INTRODUCCIÓN}

"Hay muchas maneras distintas de clasificar la cerámica. La elección depende en parte de las convenciones existentes allí donde se haga el estudio, y en parte del propósito de ese estudio. También depende del uso que otros quieran hacer de sus datos y del carácter de la colección estudiada" [1].

La cerámica ha sido uno de los elementos objeto de investigación para los estudios arqueológicos. Es por ello que en las últimas décadas se ha intensificado el estudio sobre este vestigio que da lugar a interpretaciones sobre la vida en el pasado del ser humano. "Para algunos tiene una fascinación indefinible y potencialmente contiene mucha información, que un estudio arduo y cuidadoso ha de poner de manifiesto" [1]. Para este tipo de vestigio arqueológico existen múltiples y variados temas de estudio, como lo es la forma, el estilo y la decoración. A través del estudio de la cerámica se ha entendido algunas de las dinámicas del pasado del ser humano, como su estatus social, el tipo de vida, la cosmovisión, el tipo de alimentos que consumían, etc. Es por eso que la forma y el estilo de la cerámica cumple unos intereses particulares y característicos de la cultura que la elaboró. A su vez, es importante registrar las formas y estilos de la cerámica ya que fueron utilizadas en un espacio-tiempo específico y podrían ayudar a una datación, no solo de las vasijas sino del grupo cultural que las utilizaba.

En Colombia se han realizado estudios arqueológicos referentes a la cerámica tanto de material fragmentado como de piezas completas. Este es el caso de la región del CAUCA $M E D I O$, donde habitaron, en época prehispánica, un representativo número de personas que gracias a los diferentes estudios de material cerámico, se ha podido interpretar las características del modo de vida. El desarrollo de la arqueológica en esta región ha obedecido a cuestionamientos referidos a la cerámica desde su parte tipológica y cronológica. A pesar de ello, no todo el material ha sido clasificado y estudiado de manera objetiva; aunque algunos piensen que este tipo de análisis descriptivo, formal, estilístico y de la tecnología, no se profundiza, es necesario realizar una clasificación arqueológica del material cerámico para luego desarrollar estudios específicos.

Esta investigación tiene como objetivo realizar una clasificación del material cerámico que se encuentra en $e l$ Instituto Municipal de Cultura y Fomento al Turismo (IMCFT) de la ciudad de Pereira (Risaralda), puesto que ha permanecido dentro de sus instalaciones y la única información que se tiene es un inventario y registro. Con el propósito de utilizar el material que reposa en este Instituto, el cual ha sido producto de múltiples investigaciones en Pereira (ciudad que hace parte de la región Cauca Medio), se quiere realizar una clasificación de formas y estilos de la cerámica. 
Con este estudio se espera conocer el tipo de cerámica prehispánica que tiene en custodia el Instituto Municipal de Cultura y Fomento al Turismo (IMCFT) y establecer el tipo de cerámica que se encuentra en la ciudad de Pereira (Risaralda) en la cual se determinará las formas y estilos correspondientes a las tipologías cerámicas del Cauca Medio relacionadas con diversidad de culturas en el tiempo y su distribución espacial.

\section{METODOLOGÍA}

El material arqueológico seleccionado tuvo como criterio principal que las piezas con las cuales se realizaría el estudio fueran productos de investigaciones arqueológicas en la región y no se tomaron en cuenta las que fueron el resultado de donaciones, puesto que no tienen contexto arqueológico y no se podría determinar la tipología cerámica de Pereira.

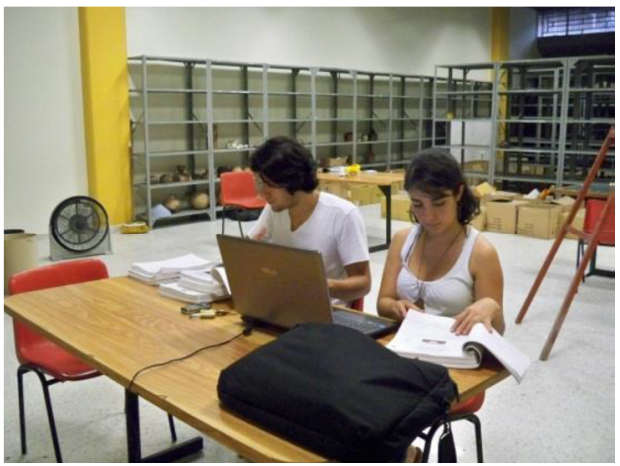

Figura 1: Selección del material arqueológico en IMCFT.

En las colecciones arqueológicas, para el Instituto Colombiano de Antropología e Historia (ICANH), se consideran como piezas completas aquellas que constituyen más del $70 \%$ de la forma original y es posible atribuirles un referente o valor histórico-cultural. Se pueden identificar piezas cerámicas como urnas, vasijas, cuencos, rodillos, volantes de huso, etc., o material lítico constituidos por azadas, hachas, manos de moler, metates etc. [2]. La colección está compuesta por 8 sub-colecciones (AKF-Autopistas del café, ALT-AltaVista, ARI-Arcoíris de la Colina, CRICiudadela Risaralda, REM-El Remanso, UTP-Universidad Tecnológica de Pereira, VIL-Villa de Leyva y VIV-Villa Verde) seleccionando 119 piezas cerámicas completas.

La clasificación de la cerámica puede obedecer a ciertos propósitos en los cuales la información quedaría con más orden y claridad para comprender cada pieza. Además permite la identificación de tipos y así reconocer la existencia de "modelos". La forma de la cerámica se estudió a partir de un sistema formal de clasificación propuesto por Gardin (1985). En la cual comparó los rasgos individuales de una vasija, como lo es la forma del cuerpo, cuello, base, asa, etc. Además, se tomó en cuenta la clasificación basada en las medidas; este método consiste en definir los tipos, de acuerdo a las dimensiones principales. Las mediciones a realizar corresponden a la altura y el diámetro de la vasija, como es el ejemplo de la clasificación realizada por Webster en 1964): "Un cuenco tiene <<una altura mayor que un tercio de su diámetro, pero menor que aquel >>; un plato, <<una altura inferior a un tercio de su diámetro, pero mayor que un séptimo del mismo >>" [1].

El estudio de la forma de la cerámica corresponde a las variables de las convenciones geométricas del cuerpo de la vasija (Labio, cuello, asa, hombro, agarradera, base, soporte), también se toma la función que la pieza represente junto con las medidas de la misma (altura, ancho y diámetro) De este modo, la forma de las vasijas cerámicas advierten a una serie de características externas como superficies, líneas y volúmenes; tamaño, complejidad y uniones de los elementos que configuran el objeto [3] . Otro aspecto de clasificación cerámica es el estilo, en este se puede identificar la característica principal de la cultura que ha realizado tal artefacto. Por ello se ha intensificado el estudio del estilo a través de la creación de tipologías con las cuales se busca crear similitudes y diferencias de la cerámica de una región. Esta creación de tipologías ayudó a distinguir la cerámica de cada grupo particular y de este modo a diferenciar el tipo de comunidades que habitaron en una región. Este tipo de estudio permite denominar "grupo cultural o simplemente cultura a un conjunto tal de rasgos asociados regularmente” [1]. De este modo, se crean grupos de estilo cerámico característico de una zona o región particular, diferenciándose de otro tipo de cultura. Para analizar el estilo de la cerámica es pertinente estudiar las siguiente características; tener en cuenta la forma, técnica de manufactura (Modelado, moldeado, enrollado, torneado, lascado, pulido, fundido y martillado), técnicas decorativas (Incisión, excisión, modelado, moldeado, acanalado, aplicación, perforación, pintura y repujado), y acabado de superficie (alisado, baño, bruñido, engobe, pegado, pulido y vidriado)

"Forma: son los códigos geométricos que definen la silueta de un recipiente o de una figura” [4]:

Formas Geométricas:
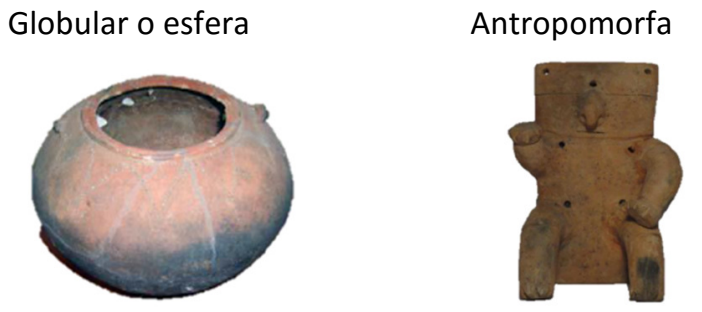

Figura 2: Algunas formas geométricas encontradas en la investigación.

La cultura material cambia en el tiempo y en el espacio y es por ello que la mayoría de los análisis arqueológicos que sobre este se realiza, tienen en cuenta el contexto en el cual se está realizando el estudio. A través de esto, se puede conocer 
los procesos tecnológicos, cronológicos, ocupacionales, etc. Por otra parte, los cambios que se identifican sobre el material hacen referencia a varias ocupaciones por parte de distintos grupos humanos u ocupaciones por el mismo grupo.

Los artefactos arqueológicos tienen un estilo específico que caracterizan a determinados grupos humanos en determinados contextos., "existen varias opciones posibles de elaborar o usar un artículo, pero la elección de algunas de ellas, dictadas por las tradiciones artesanales por parte de un grupo social son las que permiten identificar esos patrones particulares, así cada grupo social o unidad étnica posee sus patrones distintivos y coherentes, lo que percibimos en los artefactos es el estilo particular que difiere de un contexto social a otro" [5].

Estilos Cerámicos:
Cauca Medio

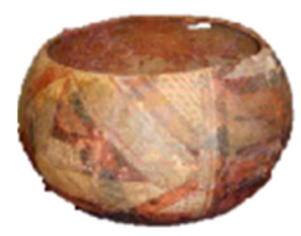

Quimbaya Tardío

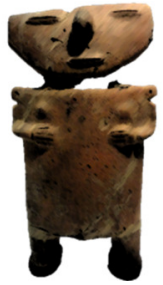

Figura 3: Algunos estilos encontrados en la investigación.

\section{ANÁLISIS DE FORMA Y ESTILO}

Las semejanzas de la forma y el estilo de la cerámica se encuentran implícitos en las piezas, es a través de ello que es posible observar una relación entre los grupos prehispánicos que las diseñaron, puesto que este vestigio permite interpretar y reconstruir un pasado que, tal vez, ha sido olvidado. Se logró establecer categorías de clasificación y con base a ello se agruparon en complejos cerámicos característicos de la región.

La colección está compuesta por 8 sub-colecciones (AKFAutopistas del café, ALT-AltaVista, ARI-Arcoíris de la Colina, CRI-Ciudadela Risaralda, REM-El Remanso, UTPUniversidad Tecnológica de Pereira, VIL-Villa de Leyva y VIV-Villa Verde) seleccionando 119 piezas cerámicas completas; en las cuales se realizó el análisis de formas y estilos para determinar el tipo de vasijas cerámicas que se encuentran en Pereira (Risaralda).

Autopistas del Café (AKF). Se analizó 88 piezas de tipo cerámico, provenientes de los proyectos de desarrollo vial armenia-Pereira-Manizales [6] y doble Calzada ArmeniaPereira-Manizales fase II [7]. Todas las piezas de esta subcolección son provenientes de investigaciones arqueológicas a cargo del antropólogo Carlos Restrepo y la mayoría fueron halladas en estructuras funerarias; ello permitió establecer las piezas que se caracterizan en esta región de Pereira.

La arqueología en Pereira se empieza a estudiar con detalle gracias a los reconocimientos arqueológicos realizados por arqueólogos de esta región, como lo es el estudio realizado por Martha Cecilia Cano en el año 2000 o Luis Gonzalo Jaramillo en 1989 [8]. Además, se inicia una expansión urbana la cual da inicio a realizar "arqueología de rescate" en los sitios en los cuales se ejecutan las construcciones de las viviendas, esto permite que se salvaguarde el patrimonio arqueológico. Desde este punto de vista, se localizó las subcolecciones ALT (Alta Vista), ARI (Arcoíris de la Colina), CRI (Ciudadela Risaralda), REM (Remanso), VIL (Villa de Leyva) y VIV (Villa Verde), pertenecientes a urbanizaciones en la ciudad de Pereira la cuales llevan el mismo nombre. Por otro lado, en las construcciones de la Universidad Tecnológica de Pereira se localizaron varias piezas las cuales llevan el código de UTP.

Formas: Las piezas cerámicas que hacen parte de investigaciones cuentan con un total de 119, clasificadas con las variables establecidas en la metodología. Las fichas diseñadas se realizaron en base a las descripciones realizadas por Rojas [4] y Adames [3].

Las formas más comunes de estas sub-colecciones son los cuerpos de forma semiglobular o semiesférica. Además, se logró identificar cuerpos de forma subglobular o esférico, globular o esfera, elipsoide horizontal, ovoide invertido, ovoide normal, cilindro, cono y figuras antropomorfas.

Semiglobular o semiesférica: Se identificó que en la subcolección $(\boldsymbol{A K F})$ hay 43 piezas, (ARI) 1 pieza, (REM) 3 piezas, (UTP) 1 pieza, y (VIV) 3 piezas, dando un total de 51 piezas.

Subglobular o esférico: Las formas de la cerámica de este tipo fueron: $(\boldsymbol{A K F}) 23$ piezas, $(\boldsymbol{A L T}) 1$ pieza, $(\boldsymbol{C} \boldsymbol{R} \boldsymbol{I}) 1$ pieza, (REM) 1 pieza, (UTP) 3 piezas, y (VIV) 2 piezas, dando un total de 31 piezas.

Globular o esfera: La cerámica de forma globular son de la sub-colección, $(\boldsymbol{A K F}) 2$.

Elipsoide horizontal: las sub-colecciones con cerámica de cuerpo elipsoide horizontal son: $(\boldsymbol{A K F}) 2$ piezas, $(\boldsymbol{R} \boldsymbol{E M}) 1$ pieza y $(\boldsymbol{V I V}) 1$ pieza, las cuales presentan un total de 4 piezas.

Ovoide normal: la sub-colección que tienen este tipo de forma es (VIV) 1 pieza.

Cilindro: las dos sub-colecciones que tienen este tipo de forma son (UTP) 1 pieza y (VIV) 1 pieza para un total de 2 piezas.

Cono: La única sub-colección que tiene la cerámica de este tipo es $(\boldsymbol{A K \boldsymbol { K }}) 3$ piezas. 
Antropomorfas: Las representaciones humanas se presentan en las sub-colecciones que la tienen: $(\boldsymbol{A K F}) 4$ piezas y $(\boldsymbol{A L T})$ 1 pieza, para un total de 5 piezas.

Los estilos que se establecieron para el material proveniente de investigaciones son el Quimbaya Tardío, Aplicado Inciso, Cauca Medio, Blanco grueso y Guavas-Buga.

Quimbaya Tardío: "El período Tardío en la historia Prehispánica del Norte de Suramérica corresponde grosso modo a los últimos 1.000 años transcurridos antes de la conquista española" [9].

Autopistas del café (AKF) hay 44 piezas, en la Sub-colección AltaVista (ALT) 2 piezas, Arcoíris de la Colina (ARI) 2 piezas, Ciudadela Risaralda (CRI) 1 pieza, Remanso (REM) 5 piezas, Universidad Tecnológica de Pereira (UTP) 3 piezas, Villa de Leyva (VIL) 1 pieza y Villa Verde (VIV) 8 piezas. Con lo anterior, se deduce que para el Período Tardío se encuentra un total 66 de piezas cerámicas que hacen parte de este estilo.

Aplicado Inciso: Este tipo de vasijas cerámicas se caracteriza por presentar técnica decorativa incisa y aplicación localizada en la parte superior. Para este estilo se identificó las siguientes piezas: sub-colección $(\boldsymbol{A K F}) 12$ piezas y $(\boldsymbol{U T P}) 1$ pieza; dando un total de 13 piezas.

Cauca Medio: "Esta cerámica se encuentra dispersa desde el norte de Manizales hasta el norte del departamento del Valle" (Bruhns 1990: 86). La decoración de este estilo cerámico es con motivos geométricos sobre un engobe naranja, en ocasiones blanco o crema. Además, algunas tienen pintura roja (asociada a tumba). Se identificó las siguientes piezas; sub-colección $(\boldsymbol{A K F}) 28$ piezas y $(\boldsymbol{U T P}) 1$ pieza, con lo cual se dio un total de 29 piezas del estilo cerámico Cauca Medio.

Blanco Grueso: Este tipo de cerámica se caracteriza por tener un engobe anaranjado, la técnica decorativa es la pintura blanca (en líneas) sobre el engobe naranja; "la pintura blanca ha sido aplicada de tal manera que las líneas sobresalen y pueden sentirse al tacto" [10]. Es poco el material que tiene este tipo de decoración, sin embargo se encontraron dos (2) piezas pertenecientes a la sub-colección $(\boldsymbol{A K F})$.

\section{CONCLUSIÓN}

El material cerámico producto de investigación, permitió identificar el tipo de cerámica que se encuentra en la ciudad de Pereira- Risaralda, el cual está relacionado con la cerámica de la región del Cauca Medio. Ello es importante puesto que reafirma la relación estrecha, observada desde la cultura material, entre las diferentes culturas que ocuparon la misma región; aclarando que la cerámica presenta sus variaciones a pesar que se localiza en la misma región, donde se puede analizar las distintas culturas.
El material cerámico está conformado por 119 piezas: en tanto la forma, Semiglobular o semiesférica 51 piezas, Globular o esfera 2 piezas, Subglobular o esférica 31 piezas, Elipsoide horizontal 4 piezas, Ovoide normal 1 piezas, Cilindro 2 piezas, Cono 3 piezas y Antropomorfa 5 piezas. De este modo, se resalta que la mayor característica de las formas encontradas en las colecciones de investigación y donación es Semiglobular o semiesférica.

Para el estilo se identificó: Cauca Medio 29 piezas, Quimbaya Tardío 66 piezas, Aplicado Inciso 13 piezas y Blanco Grueso 2 piezas.

En lo observado y analizado en esta investigación, vinculando la forma y el estilo de la cerámica que hace parte de las investigaciones arqueológicas realizadas en la ciudad de Pereira, Se estableció las formas y los estilos predominantes. La forma preponderante dentro de las colecciones producto de investigación es Semiglobular o semiesférica con un total de 61 piezas. Por otro lado, el estilo cerámico con mayor presencia dentro de estas colecciones es Quimbaya Tardío con un total de 66 piezas, seguido es el estilo Cauca Medio con 29 piezas y Aplicado Inciso con 13 piezas.

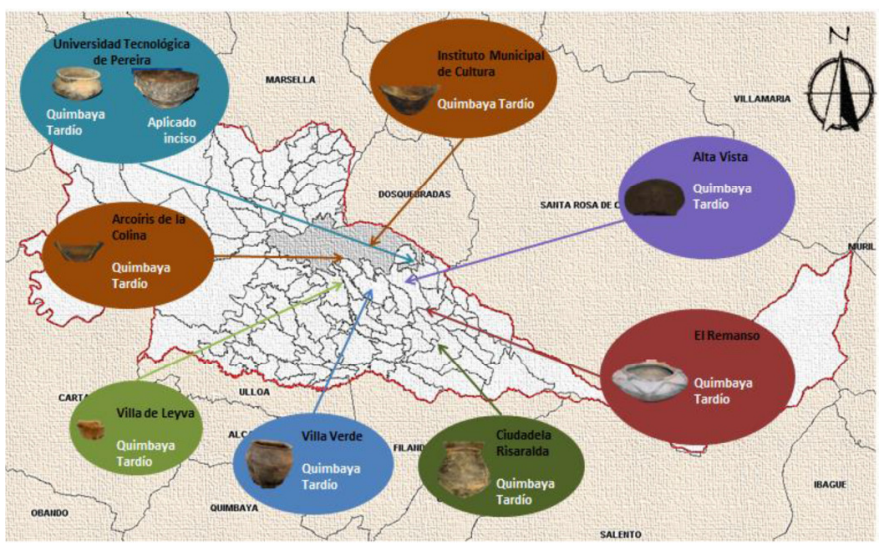

Figura 4: Formas y Estilos de la cerámica prehispánica encontrada en Pereira-Risaralda.

El trabajo arqueológico efectuado en el Instituto Municipal de Cultura y Fomento al Turismo (IMCFT), ratifica la importancia de este tipo de investigaciones. Además, se constituye como herramienta para futuras clasificaciones de la cerámica y puede servir como elemento importante a la hora de conocer la cerámica de la región del Cauca Medio.

La clasificación de la forma y el estilo de la cerámica que fue producto de investigaciones arqueológicas en la ciudad de Pereira-Risaralda, confirman que la cerámica de Pereira hace parte de la cerámica que se ubica en la región del Cauca Medio, como lo son los estilos. Cabe resaltar que la cerámica de esta ciudad presenta una pequeña variación; a pesar de tener una forma geométrica definida, su forma es un poco burda con respecto a la cerámica de otras regiones 
(comunicación personal con el antropólogo PHD Carlos Eduardo López Castaño).

Los estilos establecidos para el Cauca Medio [10], se relacionaron e identificaron con el material producto de investigaciones del IMCFT.

El estilo y la forma predominante en la cerámica producto de investigaciones es: el estilo Quimbaya Tardio y la forma Semiglobular o semiesférica.

\section{RECOMENDACIONES}

Es importante continuar con este tipo de investigación puesto que el Instituto Municipal de Cultura y Fomento al Turismo (IMCFT) posiblemente siga obteniendo más piezas arqueológicas y posiblemente no tenga ningún tipo de estudio arqueológico.

Cabe resaltar la importancia y el fomentar este tipo de estudios arqueológicos, en compilar datos de clasificación cerámica en el Cauca Medio y establecer una ficha en la cual se dé cuenta (a manera de resumen) de cada pieza que se clasifique. Es importante, también, establecer categorías de clasificación (grupos cerámicos) ara futuros trabajos arqueológicos, se insiste en la participación de varios investigadores puesto que de esta manera no solo se facilita el trabajo sino que además se corrobora la información.

\section{AGRADECIMIENTOS}

A Carlos Eduardo López Castaño, Antropólogo PDH, director de la tesis de grado la cual fue la base para esta investigación. También a mi hija Sofía Jiménez Vargas y mi futura esposa Melisa Vargas Gallego por su apoyo incondicional, fueron mi fuente de inspiración. Finalmente al laboratorio de Ecología Histórica y Patrimonio Cultural.

\section{BIBLIOGRAFÍA}

[1]. Orton, C., Tyers, P., y Vince, A., “La Cerámica en Arqueología”. Barcelona: Crítica, 1997, pp 94, 15, 180.

[2]. Álvarez, M., Bateman, Quintero, y Ramírez. "Plan de Manejo del parque Arqueológico de San Agustín, componente conservación" Bogotá: Instituto Colombiano de Antropología e Historia, 2007.

[3]. Lema, L. A. "Estructuras formales y gráficas de los volantes de huso Quimbaya”. Medellín: Colección museo universitario, Universidad de Antioquia, 1993, pp19.

[4]. Rojas, D., "Glosario para la documentación cerámica”, Santafé de Bogotá: Fondo de Promoción de la Cultura, 1993.

[5]. Sackett, J., Style, Ethnicity and Stone tools. En M. Thompson, M. T. Garcia, \& F. J. Kense (Edits.), Status, Structure and Stratification”).California: University of Calgary. 1985 pp. 277-281.

[6]. Restrepo, C., "Proyecto Desarrollo Víal ArmeniaPereira- Manizales", Autopistas del Café (20002004, 2004).

[7]. Restrepo, C., "Proyecto Desarrollo Víal Doble Calzada Armenia- Pereira- Manizales Autopistas del Café Monitoreo arqueológico Fase II". Pereira: INVIAS Autopistas del Café, 2006, Inédito.

[8]. Cano Echeverri, M. C. Reconocimientos arqueológicos en los municipios de Marsella, Dosquebradas y Pereira (Risaralda). En V. González Fernández, \& C. A. Barragán, Arqueología Preventiva en el Eje Cafetero, 2001, Bogotá: ARFO Editores e Impresores Ltda.

[9]. Rodríguez, Carlos A. "Colombia-Ecuador: 3.000 años de arte prehispánico”. Cali: Universidad del Valle, 2013, pp 110.

[10]. Bruhns, K. O. "Las culturas prehispánicas del Cauca Medio. En B. p. Fondo de promoción de la cultura, Arte de la tierra, Quimbayas" Bogotá: Presencia, 1990, pp 92.

[11]. Álvarez Morales, A. L. "Caracterización Estilística de los Volantes de Huso de Palestina-Caldas". Manizales: Tesis de Grado Universidad de Caldas, 2012.

[12]. Bennett, W. "Archaeological regions of Colombia: ceramic survey”, Yale: Yale University, 1944.

[13]. H. Trimbron, "Señorío y Barbarie en el Valle del Cauca”. Cali : Cargraphics, 2005.

[14]. V. G. Fernández, \& C. A. Barragán, "Arqueología Preventiva en el Eje Cafetero". Bogotá: ARFO editores e impresores Ltda, 2001.

[15]. Cadena Muñoz , A. M., \& Valencia García, Y. "La urna como patrón funerario en el Madalena Medio" . Manizales : Tesis de grado Universidad de caldas, 2010.

[16]. Cano Echeverri, M. C. "Investigaciones Arqueológicas en Santuario (Risaralda)”. Bogotá: Fundación de Investigaciones Arqueológicas Nacionales-Banco de la República, 1995.

[17]. Duque Gómez, L. "Los Quimbayas: Reseña etnohistórica y arqueológica”. Bogotá: Imprenta Nacional, 1970.

[18]. Fondo de promoción de la cultura. "Arte de la tierra, Quimbayas”. Bogotá: Presencia, 1990. 
[19]. Fonseca, L., "Arte de la tierra Colombia: Forma y Figura”. Bogotá: ImpreAndes S.A, 1992.

[20]. Friede, J. "Los Quimbayas: bajo la dominación española”. Bogotá: Carlos Valencia Editores, 1982.

[21]. Henao Jiménez, C. E. "Monitoreo Arqueológico Parque Temático de Flora y Fauna de Pereira”. Pereira: Licencia de intervención Arqueológica No. 1956 de 2011, 2012.

[22]. Hodder, I. "The distribution of material culture items in the Baringo District", Western Kenya. Man (N.S.), 1977.

[23]. ICANH. "Instituto Colombiano de Antropología e Historia”. Recuperado el 30 de Marzo de 2014, de www.icanh.gov.co, 2008.

[24]. Jaramillo, L. G. (2000). "Investigación arqueológica en los municipios de Chinchiná, Palestina, Villamaría y Santa Rosa de Cabal". Boletín de Arqueología. Fundación de Investigaciones Arqueológicas Nacionales (FIAN), 2000, pp29-40.

[25]. LEHPC. "Plan de manejo de la colección Arqueológica en custodia del Instituto municipal de cultura y fomento al turismo de Pereira". Pereira: Laboratorio de Ecología Histórica y Patrimonio Cultural, 2013.

[26]. López , C., Cano , M., Franco , A., \& Mora, L. (2006). "Arqueología de la cuenca del río Consota, Municipio de Pereira (Risaralda). Estudio del componente Arqueológico sitios guaduales de Canaán (66per019), Cañaveral (66per095), Matecaña y Santa Monica (66per097”). Pereira: Universidad Tecnológica de Pereira, 2006.

[27]. López Castaño, C. E., \& Aldana Sierra, F. "Reconocimiento, prospección y monitoreo arqueológico en el sitio UNICENTRO (66PER101)”, Pereira (Risaralda). Pereira, 2007.

[28]. Manrique , E. "Guía para un estudio y tratamiento de la cerámica precolombina” . Lima: concytec, 2001.

[29]. Mora González, L. M. “Análisis multitemporal de las evidencias materiales en La Catedral Nuestra Señora de la Pobreza”. Pereira: Tesis de grado Universidad de Antioquia, 2006.

[30]. Moreno , M. C. "Arqueología de Salvamento en la Vereda La Cabaña. Manizales”. Bogotá: Fundación de Investigaciones Arqueológicas Nacionales-Banco de la República, 1983.

[31]. Moreno, C., Herrera, L., \& Peña, Ó. "La historia muy antigua del municipio de Palestina (Caldas)”. Manizales: Espacio Gráfico Comunicaciones S.A, 2011.

[32]. Moreno, M. C. "Investigaciones Arqueológicas en el bajo río Guacaica, Caldas”. Bogotá: Fundación de Investigaciones Arqueológicas Nacionales-Banco de la República, 1986.
[33]. Múnera, L. C., \& Monsalve, O. "Arqueología de Rescate: Vía Alterna de la Troncal de Occidente, Sector Puente Domenicio Parma- Río Campoalegre”. Medellín: Integral, 1996.

[34]. Mora González, L. M. "Análisis multitemporal de las evidencias materiales en La Catedral Nuestra Señora de la Pobreza". Pereira: Tesis de grado Universidad de Antioquia, 2006.

[35]. Moreno , M. C. “Arqueología de Salvamento en la Vereda La Cabaña. Manizales”. Bogotá: Fundación de Invetigaciones Arqueológicas Nacionales-Banco de la República, 1983.

[36]. Moreno, C., Herrera, L., \& Peña, Ó. "La historia muy antigua del municipio de Palestina (Caldas)". Manizales: Espacio Gráfico Comunicaciones S.A, 2011.

[37]. Moreno, M. C. "Investigaciones Arqueológicas en el bajo río Guacaica, Caldas”. Bogotá: Fundación de Investigaciones Arqueológicas Nacionales-Banco de la República, 1986.

[38]. Múnera, L. C., \& Monsalve , O. "Arqueología de Rescate: Vía Alterna de la Troncal de Occidente, Sector Puente Domenicio Parma- Río Campoalegre”. Medellín: Integral, 1996.

[39]. Renfrew, C., \& Bhan, P. "Arqueología: métodos, práctica y teoría”. Barcelona, España: edisa, 2011.

[40]. Rojas, D. "Glosario para la documentación cerámica”. Santafé de Bogotá: Fondo de Promoción de la Cultura, 1993.

[41]. Silva, A. E. "Glosario para la documentación cerámica”. Santa fé de Bogotá: editorial Presencia, 1993.

[42]. Vargas Gallego, M. "La Arquitectura Prehispánica: Una Construcción Social y Espacial de la Cultura”. Manizales: Tesis de Grado en Arquitectura Universidad Nacional de Colombiasede Manizales, 2014.

[43]. Vélez Correa, F., \& Valencia Llano, A. "Caldas en las Crónicas de Indias”. Manizales: Editorial Manigraf, 2007.

[44]. Wobst, M. "Stylistic behavior and information exchange". for the director: Research Essays in honor of James B. Griffen. University of Michigan. Cleland, E.H. (ed), 1977, pp 317-342.

[45].Zuluaga, V. "Historia de Cartago la Antigua, Provincia de Popayán”. Pereira: Gráficas Buda Ltda, 2002. 